\title{
Supportive Approach to Covid-19 Treatment with Acupuncture Practice
}

\author{
Mehmet Tugrul Cabioglu1, Caner Horzum², Saltuk Cabioglu ${ }^{3}$ \\ ${ }^{1}$ Department of Physiology, Ada Kent University, Istanbul, Turkey \\ ${ }^{2}$ Acupuncture Education Coordinating Center, Dr. Abdurrahman Yurtaslan Ankara Oncology Training and Research Hospital, \\ University of Health Sciences, Ankara, Turkey \\ ${ }^{3}$ Emergency Department, Murat Kölük State Hospital, Istanbul, Turkey \\ Email: tugcab@gmail.com, canerhorzum@gmail.com, saltukcab@hotmail.com
}

How to cite this paper: Cabioglu, M.T., Horzum, C. and Cabioglu, S. (2020) Supportive Approach to Covid-19 Treatment with Acupuncture Practice. Open Access Library Journal, 7: e6457.

https://doi.org/10.4236/oalib.1106457

Received: May 24, 2020

Accepted: December 28, 2020

Published: December 31, 2020

Copyright (๑) 2020 by author(s) and Open Access Library Inc.

This work is licensed under the Creative Commons Attribution International License (CC BY 4.0).

http://creativecommons.org/licenses/by/4.0/

\section{(c) (i) Open Access}

\begin{abstract}
Nowadays, there is no specific antiviral treatment for COVID-19 that has been proven to be safe and effective. Therefore, the prophylactic approach, that is, the preventive approach, especially in risk groups, comes plan to the fore before the disease occurs. In the Covid-19 pandemic, the preventive prophylactic approach becomes even more important as there are doctors, nurses, healthcare professionals and public officials working in this period, especially within the risk group. It is known that acupuncture administration causes an immunomodulating effect. There are many studies on this subject, especially in recent years. We classified the Covid-19 infection according to the stages of development and syndromes, considering Traditional Chinese Medicine. In this review, we discussed at which stage and syndrome acupuncture application can be effective, especially prophylaxis.
\end{abstract}

\section{Subject Areas}

Infectious Diseases, Internal Medicine

\section{Keywords}

Covid-19, Acupuncture, Prophylaxis, Immunomodulation, Antiviral Treatment

\section{Introduction}

Coronaviruses (Cov) are a family of viruses that cause more serious illnesses from the common cold, such as Middle East Respiratory Syndrome (MERS-CoV) and Severe Acute Respiratory Syndrome (SARS-CoV). As a result of comprehensive researches, it has been revealed that SARS-CoV is transmitted from 
musk cats and MERS-CoV from single humped camels to humans. There are many coronoviruses types that have not been transmitted to humans yet, but are detected in animals [1] [2].

The factors that cause flu are Influenza A and Influenza B viruses. It is the Influenza A virus that causes common disease in humans. Influenza A virus causes disease in humans and animals like pigs, birds and horses; influenza B only in humans. There are also subtypes of influenza A virus that causes disease only in animals. The best known example of this is bird flu. This virus has been shown to be also transmitted to humans in recent years [3].

Covid-19 falls under the category of "epidemic" disease. The effect of the climate in Wuhan on the spread of Covid-19 can be understood using Traditional Chinese Medicine theories. Chinese medicine academia and many Chinese medicine researchers consider that the cold and dampness, disrupting especially the yang qi, caused by the constant rainy and abnormal hot climate in Wuhan in December 2019, and, paved the way for the epidemic. Patients were mostly affected by dampness-cold pathogens that affect lung and spleen [4] [5].

If we approach Covid-19 viral infection in terms of Traditional Medicine Diagnoses [6] [7] [8] [9];

1) Wind-Cold Syndrome (Early period-1)

2) Damp-Cold Syndrome (Early period-2)

3) Heat Attacking the Lung (Intermediate period-1)

4) Damp-Heat Attacking the Lung (Intermediate period-2)

5) Blockage in Lung and visceral organs (Severe period-Intensive care unit)

6) Qi deficiency in Lung and Spleen (Recovery period)

However recently, the prophylactic approach that is the preventive approach before the disease occurs has come to the fore especially in risky groups [10]. In the Covid-19 pandemic, the preventively prophylactic approach becomes even more important, as in the risky groups there are doctors, nurses, healthcare professionals and public officials in charge during this period.

\section{Methods}

\subsection{Prophylaxis in Covid-19 Treatment}

1) Due to contamination over the eyes, mouth and nasal mucosa [11] [12], local acupuncture points related to these structures, around the nose, Bitong, LI 20 and Yintang, around the mouth, ST 4, DU 26 and around the eye, ST 1, UB 2 useable [6]. Using these points, by pricking the needle, in these contamination areas, creating microinflammation, cytokines such as TGF- $\alpha$, TGF- $\beta$ released from platelets in these regions, attract neutrophils to the region. During this period, we see that tissue macrophages are also active. After a while, it activates in other immune cells throughout the body. So when there is a viral infection in these areas, immune cells are ready to respond [13].

2) Respiratory transmission and initial exposure tonsilla, pharynx, trachea and owing to then it is in the lungs, we use the Yuan (Source) point LU 9 and 
Luo-connecting point LU 7 of the lung meridian.

3) Immunomodulatory points, LI4, ST36, SP6, REN 12, REN 6 points are used [13].

Purpose of acupuncture application in the medical observation stage; Motivating the antipathogenic qi of the human body, the functions of the lung and spleen and to distribute epidemic pathogens to strengthen the defense capacity of internal organs [14]. It has been reported in recent studies that the application to ST 36 acupuncture point with anti-inflammatory and anti-allergic properties is the most commonly used acupuncture point for immunomodulation [15]. Many studies provide evidence suggesting that acupuncture application to LI 4 , SP 6 and REN 6 points, especially ST 36, causes a significant increase in natural killer (NK) cell activity [16]. In a controlled laboratory experiment, Hubei Chinese Medicine University researchers discovered that acupuncture point ST 36 (Zusanli) electroacupuncture stimulation "increases the level of immune cytokines and spleen CD4 $+\mathrm{T}$ cells through TRPV channels". Findings are important because $\mathrm{CD} 4+\mathrm{T}$ cells are very important in human immune systems. These cells are called helper $\mathrm{T}$ cells and are active in the immune system's defense against microorganisms, including viruses. CD4 + T cells are capable of differentiating into many different cell subsets in activating the body's immune system cells, immunoregulation and cytotoxic defense mechanisms. Application to ST 36 acupuncture point is effective in activating the immune system [17].

A large amount of porphyrin is needed for the virus to survive. New coronavirus attacks hemoglobin in erythrocytes and exposes porphyrin. Based on low levels of heme in coronavirus patients; It is considered that the virus binds directly with human hemoglobin. Porphyrin is an important material for heme synthesis. It is thought that the virus may be competing with iron for porphyrin as the cause of iron increase in the body [18]. Considering this physiopathology, we can add the Sp 10 (Sea of Blood) point used in Heat in the Blood syndrome to prophylaxis in blood related problems [19].

\subsection{Covid-19 Treatment Stages}

1) Wind-Cold Sendrome [8]

Clinical Findings: Heat (mostly low-grade fever), feeling uncomfortable with cold, chills, headache, discomfort from the throat, pain in the muscles.

Tongue Findings: Pale, coating is white and thin.

Pulse Findings: Weak and floating pulse.

Treatment Strategy: Wind and cold elimination.

Treatment: LU 9, LU 7, UB 12, UB 13, GB 20.

Note: Radiological findings of this period in current medicine, there is no finding in tomography.

2) Damp-Cold Sendrome [9]

Clinical Findings: Cough, tension in the chest, moderate difficulty breathing, weakness, decreased appetite, nausea, vomiting, swelling in the abdomen, diarrhea or constipation. 
Tongue Findings: Pale tongue, adhesion and partially white coating on the tongue.

Pulse Findings: Weak and slippery pulse.

Treatment Strategy: Deactivation of humidity and cold.

Treatment: LU 9, LU 7, UB 12, UB 13, GB 20, ST 36, ST 40, SP 9.

For Lung Strengthening (Tonification); LU 9, LU 7, UB 13.

To Inactivate Damp; ST 36, ST 40, SP 9.

3) Heat Attacking the Lung [8]

Clinical Findings: Heat, discomfort from the cold, dry cough, insufficient phlegm, pain and painful muscles in the limbs, weakness, headache.

Tongue Findings: Trunk and tip are red, yellow coating on it.

Pulse Findings: Rapid pulse.

Treatment Strategy: Deactivating heat from the lung.

Treatment: LU 9, LU 7, UB 13, UB 14, UB 15, LI 4, LI 11, DU 14.

For Lung Strengthening (Tonification); LU 9, LU 7, UB 13, UB 14, UB 15.

To Disable Heat: LI 4, LI 11, DU 14.

Note: Radiological findings of this period in current medicine, tomography shows scattered ground glass opacity in both lungs.

4) Damp-Heat Attacking the Lung [8]

Clinical Findings: Heat, advanced shortness of breath, dry cough, having sputum but having difficulty removing sputum, general pains in the body, headache, weakness, fatigue, recumbency want, nausea and vomiting, diarrhea.

Tongue Findings: Trunk and tip red, sticky and yellow coating on the tongue, tooth marks on the edge of the tongue.

Pulse Findings: Rapid and slippery pulse.

Treatment Strategy: Inactivation of damp and heat from the lung.

Treatment: LU 9, LU 7, LU 10, LI 4, LI 11, DU 14, ST 36, ST 40, SP 9.

For Lung Strengthening (Tonification); LU 9, LU 10, LU 7.

To Disable Heat; LI 4, LI 11, DU 14.

To Inactivate Damp; ST 36, ST 40, SP 9.

Note: Radiological findings of this period in current medicine, tomography shows that both lungs have a glass opacity in more than one scattered or large area.

5) Blockage-Collapse in the Lungs and Visceral Organs [8] [Patient Group to be treated in intensive care $]^{*}$

Clinical Findings: Very severe breathlessness and as a result a feeling of suffocation, wheezing, increased breathing frequency (These patients can only maintain their breathing with a respiratory support device).

Tongue Findings; Dark purplish tongue, yellow dry tongue coating or thick and oily yellow coating.

Pulse Findings; Rapid and slippery pulse.

Urine: Red colored urine (erythrocytes in the urine).

*Treatment Strategy: In this period, we do not think that acupuncture will be effective because the patients are connected to the respiratory device and 
the lungs are under the control of the device.

Note: Radiological findings of this period in current medicine, tomography shows glass opacity in more than one large area in both lungs.

6) Lung and Spleen Qi Deficiency [9] [20] [Recovery Period]

Clinical Findings: Fatigue, anorexia, nausea, abdominal distention and fullness, constipation or loose sticky stool.

Tongue Findings: A swollen tongue, white and sticky coating on it.

Pulse Findings: Deep and slow pulse.

Treatment Strategy: Strengthening the Qi, lung and spleen [6].

Treatment: LU 9, LU 7, UB 12, UB 13, ST 36, ST 40, SP 9.

For Lung Strengthening (Tonification); LU 9, LU 7, UB 13.

Strengthening the Spleen; ST 36, ST 40, SP 9.

\section{Conclusions}

There is no specific antiviral therapy or vaccine for Covid-19. Studies on this subject continue. Clinical treatment of Covid-19 has so far been limited to support and palliative care. Also, Covid-19 mutated and virulence potent, a new virus can cause a pandemic again. Therefore, immunomodulation has gained importance in prophylaxis that we will perform with acupuncture application.

Acupuncture application, in the light of the many literatures we have read on this subject, especially prophylaxis, effective in the initial stages of Wind-Cold Syndrome, Damp-Cold Affecting the Lung and Lung and Spleen Qi Deficiency (Recovery Period) syndromes; Heat Attacking the Lung, Damp-Heat Affecting Lung syndromes will contribute to plants, medicines and other treatments. In the Lung and Visceral Organs Blockage-Collapse, this treatment will need to be done in intensive care, we think that acupuncture application will not be necessary.

\section{Conflicts of Interest}

The authors declare no conflicts of interest regarding the publication of this paper.

\section{References}

[1] Cui, H.T., Li, Y.T., Guo, L.Y., Liu, X.G., Wang, L.S., et al. (2020) Traditional Chinese Medicine for Treatment of Coronavirus Disease 2019: A Review. Traditional Chinese Medicine, 2, 65-73.

[2] Wit De, E., Van Doremalen, N., Falzarano, D. and Munster, V.J. (2016) SARS and MERS: Recent Insights into Emerging Coronaviruses. Nature Reviews Microbiology, 14, 523-534. https://doi.org/10.1038/nrmicro.2016.81

[3] Boktor, S.W. and Hafner, J.W. (2020) Influenza. In: StatPearls [Internet], StatPearls Publishing, Treasure Island, Treasure, 1-19. https://www.ncbi.nlm.nih.gov/books/NBK459363/

[4] Ma, Y., Zhao, Y., Liu, J., He, X., Wang, B., Fu, S., Yan, J., et al. (2020) Effects of Temperature Variation and Humidity on the Death of COVID-19 in Wuhan, Chi- 
na. Science of the Total Environment, 724, Article ID: 138226. https://doi.org/10.1016/j.scitotenv.2020.138226

[5] Sajadi, M.M., Habibzadeh, P., Vintzileos, A., et al. (2020) Temperature, Humidity, and Latitude Analysis to Predict Potential Spread and Seasonality for COVID-19.

https://ssrn.com/abstract $=3550308$

https://doi.org/10.2139/ssrn.3550308

[6] Cheng, X.N. (2016) Chinese Acupuncture and Moxibustion. Foreign Languages Press, Beijing, 295-315.

[7] Maciocia, G. (2008) The Practice of Chinese Medicine the Treatment of Disease with Acupuncture and Chinese Herbs. Second Edition, Churchill Livingstone, Edinburgh, 1313-1337.

[8] Chen, J.K. (2020) How COVID-19 (2019-nCoV) Is Currently Treated in China with TCM. Lotus Institute of Integrative Medicine, 1-13.

https://www.elotus.org/article/how-covid-19-2019-ncov-currently-treated-china-tc $\underline{\mathrm{m}}$

[9] Sun, P. and Zhou, W.S. (2020) Acupuncture in the Treatment of COVID-19: An Exploratory Study. Journal of Chinese Medicine, 123, 14-20.

[10] Doss, M. (2020) Treatment of COVID-19 with Individualized Immune Boosting Interventions. OSF Preprints. https://doi.org/10.31219/osf.io/6zy9u

[11] Meng, L., Hua, F. and Bian, Z. (2020) Coronavirus Disease 2019 (COVID-19): Emerging and Future Challenges for Dental and Oral Medicine. Journal of Dental Research, 99, 481-487. https://doi.org/10.1177/0022034520914246

[12] Lai, T.H.T., Tang, E.W.H., Fung, K.S.C. and Li, K.K.W. (2020) Reply to "Does Hand Hygiene Reduce SARS-CoV-2 Transmission? Graefe's Archive for Clinical and EXperimental Ophthalmology, 258, 1135. https://doi.org/10.1007/s00417-020-04653-4

[13] Cabioğlu, M.T. and Cetin, B.E. (2008) Acupuncture and Immunomodulation. The American Journal of Chinese Medicine, 36, 25-36. https://doi.org/10.1142/S0192415X08005552

[14] Liu, W., Guo, S., Wang, F. and Hao, Y. (2020) Understanding of Guidance for Acupuncture and Moxibustion Interventions on COVID-19 (Second Edition) Issued by CAAM. World Journal of Acupuncture-Moxibustion, 30, 1-4. https://doi.org/10.1016/j.wjam.2020.03.005

[15] Wang, Z., Chen, T. and Long, M. (2017) Electro-Acupuncture at Acupoint ST36 Ameliorates Inflammation and Regulates Th1/Th2 Balance in Delayed-Type Hypersensitivity. Inflammation, 40, 422-434. https://doi.org/10.1007/s10753-016-0487-Z

[16] Johnston, M.F., Ortiz Sanchez, E., Vujanovic, N.L. and Li, W. (2011) Acupuncture May Stimulate Anticancer Immunity via Activation of Natural Killer Cells. Evidence-Based Complementary and Alternative Medicine, 2011, Article ID: 481625. https://doi.org/10.1093/ecam/nep236

[17] Healthcare Medicine Institute (2017) Acupuncture İmmune System Discovery. https://www.healthcmi.com/Acupuncture-Continuing-Education-News/1803

[18] Liu, W. and Li, H. (2020) COVID-19: Attacks the 1-Beta Chain of Hemoglobin and Captures the Porphyrin to Inhibit Human Heme Metabolism. https://doi.org/10.26434/chemrxiv.11938173.v7

[19] Yin, G. and Liu, Z. (2000) Advanced Modern Chinese Acupuncture Therapy. First Edition. New World Press, Beijing, 447.

[20] World Federation of Acupuncture and Moxibustion Societies (WFAS) (2020) Guidelines on Acupuncture and Moxibustion Intervention for COVID-19 (Second Edition). 